\section{$\underset{\substack{\text { hommes } \\ \text { \& migrations }}}{ }$}

\section{Hommes \& migrations}

Revue française de référence sur les dynamiques

migratoires

$1311 \mid 2015$

Femmes et migrations

\title{
Nadia Agsous. Des hommes et leurs mondes. Entretien avec Smaïn Laacher
}

Alger, Dalimen 2014, 212 pages, 600 DA.

\section{Mustapha Harzoune}

\section{(2) OpenEdition}

Journals

Édition électronique

URL : http://journals.openedition.org/hommesmigrations/3403

DOI : 10.4000/hommesmigrations.3403

ISSN : 2262-3353

Éditeur

Musée national de l'histoire de l'immigration

Édition imprimée

Date de publication : 1 juillet 2015

Pagination : 196-197

ISBN : 978-2-919040-32-2

ISSN : 1142-852X

Référence électronique

Mustapha Harzoune, "Nadia Agsous. Des hommes et leurs mondes. Entretien avec Smaïn Laacher», Hommes \& migrations [En ligne], 1311 | 2015, mis en ligne le 09 février 2016, consulté le 24 septembre 2020. URL : http://journals.openedition.org/hommesmigrations/3403 ; DOI : https://doi.org/10.4000/ hommesmigrations.3403 


\section{LIVRES}

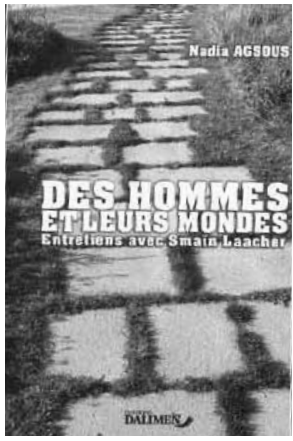

\section{Nadia Agsous}

Des hommes

et leurs mondes.

Entretien avec Smaïn

Laacher

Alger, Dalimen, 2014,

212 pages, $600 \mathrm{DA}$

Nadia Agsous propose neuf entretiens où le sociologue évoque, à travers ses livres et recherches, l'immigration, les figures du "clandestin" et du "sans-papiers", les violences faites aux femmes, comme migrantes mais aussi dans l'espace de la famille, l'école ou les révoltes dites "arabes". Davantage que les enseignements d'une recherche qui congédient les fausses perceptions médiatico-politiques, ces entretiens font goûter le sel, la quintessence d'un intellectuel actif, attentif et solidaire.

Tout tient peut-être à une sensibilité : Smaïn Laacher n'est pas un Platon égaré dans les champs de la socio. Chez lui, les hommes et les femmes ne sont pas une Idée ou un Concept. Ils sont de chair et d'os, de volitions et de contraintes, d'élans et de contradictions. "Ce ne sont pas les controverses intellectuelles ultrasophistiquées qui m'intéressent. Mon objectif est d'examiner des épreuves au plus près des pratiques des personnes et la manière dont celles-ci y font face." De ce qui n'est autre qu'une façon d'être au monde “j'atteste qu'il n'y a d'Etre humain/que Celui dont le coeur tremble d'amour/pour tous ses frères en humanité", écrit le poète Abdellatif Laâbi) - procède une pensée qui fait de Smaïn Laacher un stimulant sociologue des épreuves qui, sans tourner le dos aux rapports de domi- nation, se recentre "sur l'expérience des personnes. (...) Et forcément cela nécessite de revisiter tous les discours dominants sur l'immigration et l'immigré(e)". Exit alors les poncifs populistes, misérabilistes, les facilités et impasses sémantiques au profit d'analyses dynamiques, contrastées, intégrant la complexité de toute existence. Ainsi, l'école - "l'épreuve de la séparation d'avec les siens" - pour être l'espace de la reproduction sociale et de l'échec est aussi "une arme au service des dominés". Ne serait-ce que parce qu'elle "fournit des armes dans cet effort de compréhension de soi, des autres et du monde. Elle permet la réduction de l'incertitude par l'élucidation".

Ce livre révèle un intellectuel rigoureux - cela devrait faire partie du paquetage - mais aussi courageux. Aucunes circonvolution ici quand il parle du racisme en Algérie ou au Maroc, du peu de cas que font ces États du droit et de la protection des personnes, de l'état de la recherche au Maghreb ou de "I'incapacité" des Algériens d'"aborder les contradictions et de les résoudre pacifiquement" ou d"sunifier autour d'une cause nationale". Idem sur la question religieuse: "Dans la société d'accueil, la religion ne doit pas régir les rapports entre hommes et femmes et ne doit en aucun cas porter atteinte à la volonté et à l'autonomie des femmes", y compris en leur laissant la liberté de "choisir leur mari en dehors de la confession musulmane". Idem à propos de ces jeunes de banlieue gagnés par le racisme, l'antisémitisme ou des attitudes fascisantes. La sociologie de Smaïn Laacher, pugnace, vigilante, stimulante, porte sur des populations "engagées dans des pro- 
cessus de confrontation à de nouveaux mondes ou à des univers en transition". Émergences donc de nouvelles pratiques, de nouveaux cadres, de nouvelles obligations morales et cognitives, émergence du nouveau sur ou avec de l'ancien. Les champs d'étude foisonnent : rapport entre État, nation et individus, entre nation et présence étrangère (éclairé via les notions de "confiance, de défiance et de défi"), entre frontière et immigration, identités et territoires, les interactions entre les structures familiales et les mécanismes de reproduction des logiques de domination, l'État de droit où "la démocratie demeurera un vain mot" aussi longtemps que la question de la liberté de la femme ne sera pas posée.

Mustapha Harzoune

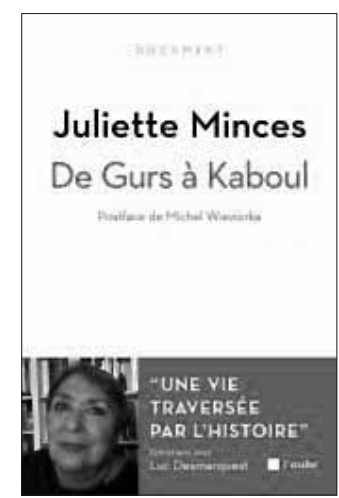

Juliette Minces

De Gurs à Kaboul. Entretien avec Luc Desmarquest.

Postface de Michel Wieviorka, La Tour d'Aigues, édition de l'Aube, 2015, 320 pages, $20 €$.

Juliette Minces fit partie durant de nombreuses années du comité de rédaction de la revue. Une fois n'est pas coutume, nous évoquerons le livre si ce n'est d'une amie à tout le moins d'une relation à la présence toujours amicale et souriante. Cette proximité ne sera pas complicité, même si ces entretiens sont rares. Rares parce que Juliette Minces parle avec une franchise qui ne court plus les rues. Ses propos sont sans arrière-pensées. Pas de double fond ici. Elle ne soupèse pas ses mots au trébuchet d'une carrière ou d'une posture, d'un rapport de séduction ou d'intérêt. C'est en toute sincérité qu'elle évoque son enfance, sa famille, Ménilmontant, son parcours de fille unique d'un couple d'émigrés juifs polonais, l'internement, avec Esther, sa mère, dans le camp de Gurs. Ensuite viendra le temps des engagements : de l'indépendance algérienne à la défense des femmes afghanes en passant par la cause kurde. Juliette Minces ne cache pas ses fragilités, Gurs et ses séquelles, son sentiment d'illégitimité, sa peur de d'abandon, ses fantômes, ses moments de dépression aussi, jusqu'à ses "échecs": "J'ai construit ma maison sur des dunes mouvantes." Aujourd'hui il y a les épreuves de l'âge, la "solitude" et le "trop tard" confié "le cœur serré".

Sociologue, franc-tireur de la pensée française, Juliette Minces a écrit et enseigné sur le monde ouvrier, l'immigration, l'islam, le féminisme. Par convictions, héritées en partie de ses parents communistes, et par choix, elle a toujours été aux côtés de l'humanité souffrante. En toute liberté : loin des appareils, des carcans idéologiques et des gratifications institutionnelles. Libre donc! En refusant de faire joujou avec les sirènes du paternalisme et de la victimisation, cette liberté elle ne la refusa pas non plus aux immigré(e)s. C'est aussi pourquoi, à l'Idée de l'immigré, elle a toujours 
préféré l'immigré. En chair et en os. À la "discourite" (dixit Driss Chraïbi), elle préfère le "goût du concret", "rendre la réalité palpable", "donner la parole à mes interlocuteurs". Et comme "le monde est une branloire pérenne", sa pensée est restée, là aussi, libre. Toujours vigilante. Ainsi, en ces temps de mondialisation, elle invite encore "à réfléchir différemment, y compris aux rapports sociaux, au lieu de rester cloué par des habitudes de pensée qui remontent au XIX siècle".

C'est à Gurs, parmi les détenus de nationalités diverses, qu'elle fait l'apprentissage de l'internationalisme ou du cosmopolitisme. Cette expérience donnera, dans le feu des engagements futurs, un viatique et une éthique : "J'ai découvert combien les besoins des êtres humains étaient universels, malgré des cultures différentes et des religions étouffantes. C'est pourquoi je ne suis pas tombée dans le culturalisme quand j'ai commencé à travailler sur l'immigra- tion." D'ailleurs, "je crois qu'on crée des problèmes psychologiques quand on traite les gens différemment, en insistant sur les différences plutôt que sur les similitudes". Cela commence sur les bancs et les cantines de l'école publique.

C'est aussi à Gurs que naît la colère inentamée: "Trop de choses me rappellent le passé, et pas le meilleur : qu'il y ait aujourd'hui encore des camps de rétention où on regroupe des gens avec des enfants, des expulsions sans véritables raisons, un racisme accru, des amalgames dangereux concernant immigrés, réfugiés, demandeurs d'asile... Le taux de chômage et la richesse insolente de certains me révulsent, mais je n'agis plus. Pourtant, ayant séjourné à Gurs, je devrais être plus active. Mais je n'en ai plus la force." Ici, loin de s'opposer, éthique de conviction et éthique de responsabilité se complètent et se renforcent. Voilà aussi pourquoi ces entretiens sont rares.

M. H.

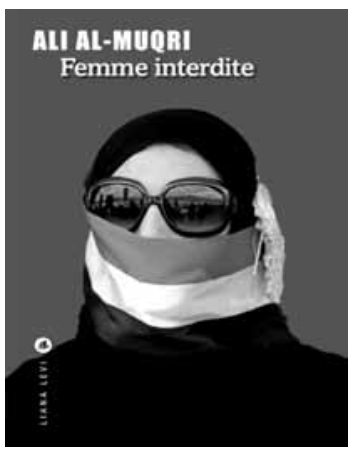

\section{Ali al-Muqri}

Femme interdite

traduit de l'arabe (Yémen) par Khaled Osman, en collaboration avec Ola Mehanna, Paris, Liana Levi, 2015, 200 pages, $19 €$.

L'imam IzzatAl-Attiyahet son collègue d'Al-Azhar Abd El-Mahdi Abd ElKader ont emballé les réseaux sociaux avec une fatwa qui recommanderait aux femmes, pour ne plus avoir à porter un voile lorsqu'elles partagent seule à seul un espace de travail avec un collègue masculin, d'allaiter cinq fois ledit collègue. Cinq anodines tétées et tombe le voile ! Explication : donner le sein "est considéré comme un acte maternel qui empêcherait tout acte sexuel". Ben voyons! Exit après ça le désir lubrique des obsédés prédateurs à moustache (le port du voile l'insinue), impuissants ou éjaculateurs précoces (Ali al-Muqri l'affirme). 
Cette pacifique fatwa illustre un propos de Femme interdite qui dénonce les stupidités auxquelles conduit une organisation sociale régie par une obsession: "Les femmes sont des goules, ce sont elles qui séduisent les hommes. Sans elles, ils seraient la pureté même." De sorte qu'il faut, dès l'âge de neuf ans, dissimuler les corps, des pieds à la tête, couper le son - "la voix de la femme est indécence" -, traquer l'équivoque jusqu'aux prénoms - qui pourrait exciter le désir de nos gugusses. "Femmedéfendue", "interdite”! Mais, pour devoir déserter les corps, le sexe n'en occupe pas moins tous les esprits, de sorte que, en ces temps de formalisme et de bigoterie, les "principes droits de l'islam" condamnent l'abstème à ingurgiter de drôles de bibines. Comme cette fatwa suscitée, resucée par Ali al-Muqri pour l'habitacle d'un taxi. Comme ces cours sur le licite et l'illicite en matière de "conjonction" prodigués par écran interposé : pour ne pas être en présence physique de ses étudiantes, le professeur n'en est pas moins émoustillé par sa suggestive leçon. En poussant la logique à son extrême, en utilisant l'ironie comme méthode de dévoilement, Ali al-Muqri montre les aberrations d'une codification des comportements reposant sur une séparation formaliste, sans âme, entre licite et illicite, hallal et haram. Sauf qu'ici ce n'est pas pile ou face mais plutôt : "pile je gagne, face tu perds" ! Tant les règles sont absurdes et le jeu cruel.

Femme interdite raconte l'éducation sentimentale ou plutôt sexuelle contrariée d'une jeune Yéménite. Ses aînés,
Loula,lasœur,etRaqib,lefrère,contestent l'ordre ambiant : libération par le vagin pour l'une, par le marxisme pour l'autre. Loula et Raqib morigènent leur cadette. Via des "cassettes culturelles" où des hommes et des femmes s'adonnent au "charivari" (en un mot du porno) pour l'une, via Rosa Luxemburg pour l'autre. Si Loula se montre constante, son marxiste de frère, après avoir convolé, ira fricoter du côté du salafisme. L'égarement individuel comme miroir des impasses du collectif. La cadette, victime d'une injonction paradoxale, poursuit un rigoureux cursus théologico-scolaire tout en restant excitée par les "cassettes culturelles", travaillée par de puissantes pulsions sexuelles qui jamais ne seront satisfaites! "Comment trouver la paix intérieure quand on est consumé par les feux du désir, par son volcan qui ne connait pas de rémission ?", demande la jeune femme. Elle finira par rechercher la "paix intérieure" chez ce voisin qui lui a offert un enregistrement de la chanson "Questionnez mon coeur" ("Salou qalbi") d'Oum Kalthoum. Le poème, communément perçu comme religieux, sert de trame au roman qui laisse alors deviner un tout autre sens.

Femme interdite est un roman (et une langue) drôle,érotique, savant; une sociologie, brutale parfois mais efficace, sur les aspirations et les tentatives de la fratrie pour s'extraire de ce cercle meurtrier du hallal et du haram, du pile ou face. Le frangin délaissera les frérots pour explorer une autre route. Quant aux deux sœurs...

M. H. 